\title{
Nanoencapsulation of natural triterpenoid celastrol for prostate cancer treatment
}

\author{
This article was published in the following Dove Press journal: \\ International Journal of Nanomedicine \\ 30 October 2015 \\ Number of times this article has been viewed
}

\author{
Vanna Sanna ${ }^{1,2}$ \\ Jean Christopher \\ Chamcheu $^{3}$ \\ Nicolino Pala' \\ Hasan Mukhtar ${ }^{3}$ \\ Mario Sechi ${ }^{1,2}$ \\ Imtiaz Ahmad Siddiqui ${ }^{3}$ \\ 'Department of Chemistry and \\ Pharmacy, University of Sassari, \\ Sassari, Italy; ${ }^{2}$ Laboratory of \\ Nanomedicine, University of Sassari, \\ Sassari, Italy; ${ }^{3}$ Department of \\ Dermatology, University of Wisconsin, \\ Madison, WI, USA
}

Correspondence: Vanna Sanna Department of Chemistry and Pharmacy, Laboratory of Nanomedicine, University of Sassari, Via Vienna 2, 07I00, Sassari, Italy

Tel +39079998619

Fax +39079 228720

Email vsanna@uniss.it

Imtiaz Ahmad Siddiqui

Department of Dermatology, Medical

Sciences Center, University of

Wisconsin - Madison, 1300 University

Avenue, Madison, WI 53706, USA

Tel + I 6082626389

Fax + I 6082635223

Email iasiddiqui@wisc.edu

\begin{abstract}
Celastrol (CL), a triterpenoid extracted from the Chinese herb Tripterygium wilfordii, has recently attracted interest for its potential antitumor effects. However, unfavorable physicochemical and pharmacokinetics properties such as low solubility, poor bioavailability, and systemic toxicity, are limiting its therapeutic application. In this context, the development of innovative nanocarriers can be useful to overcome these issues, and nanoencapsulation would represent a powerful strategy. In this study, we developed novel CL-loaded poly( $\varepsilon$-caprolactone) nanoparticles (NPs), and investigated their antiproliferative efficacy on prostate cancer cells. CL-NPs were prepared using a nanoprecipitation method and fully characterized by physicochemical techniques. The antiproliferative effects on LNCaP, DU-145, and PC3 cell lines of CL-NPs, compared to those of free CL at different concentrations $(0.5,1.0$, and $2.0 \mu \mathrm{M})$, were investigated. Moreover, fluorescence microscopy was utilized to examine the cellular uptake of the nanosystems. Furthermore, to elucidate impact of nanoencapsulation on the mechanism of action, Western analyses were conducted to explore apoptosis, migration, proliferation, and angiogenesis alteration of prostate cancer cells. The results confirmed that CL-NPs inhibit proliferation dose dependently in all prostate cancer cells, with inhibitory concentration ${ }_{50}$ less than $2 \mu \mathrm{M}$. In particular, the NPs significantly increased cytotoxicity at lower/medium dose $(0.5$ and $1.0 \mu \mathrm{M})$ on DU145 and PC3 cell lines with respect to free CL, with modulation of apoptotic and cell cycle machinery proteins. To date, this represents the first report on the development of biocompatible polymeric NPs encapsulating CL. Our findings offer new perspectives for the exploitation of developed CL-NPs as suitable prototypes for prostate cancer treatment.
\end{abstract}

Keywords: celastrol, tripterine, nanoparticles, poly( $\varepsilon$-caprolactone), prostate cancer

\section{Introduction}

Celastrol (CL), also known as tripterine, a major biologically active pentacyclic triterpenoid compound (Figure 1) isolated from the traditional Chinese medicinal herb Tripterygium wilfordii, has been used for centuries in the treatment of autoimmune disease, ${ }^{1}$ asthma, ${ }^{2}$ chronic inflammation, ${ }^{3}$ and neurodegenerative disease. ${ }^{4}$ In recent years, it has attracted interest for its potential antitumor effects against leukemia, ${ }^{5}$ glioma, ${ }^{6}$ breast, ${ }^{7,8}$ and prostate cancer cells. ${ }^{9}$

Focusing on prostate cancer, CL has been proposed as a promising medicinal small molecule for hormone-refractory prostate neoplasia. Previously, Yang et al first reported that CL directly inhibits the chymotrypsin-like activity of proteasome in androgen-independent PC3 (androgen receptor [AR]-negative) and androgen-dependent LNCaP (AR-positive) prostate tumor cells, accompanied by suppression of AR protein expression, and induction of apoptosis. ${ }^{9}$ More recently, Dai et al demonstrated that CL suppresses androgen-independent prostate cancer proliferation, invasion, and angiogenesis by inducing the apoptotic machinery and attenuating constitutive NF-kB 


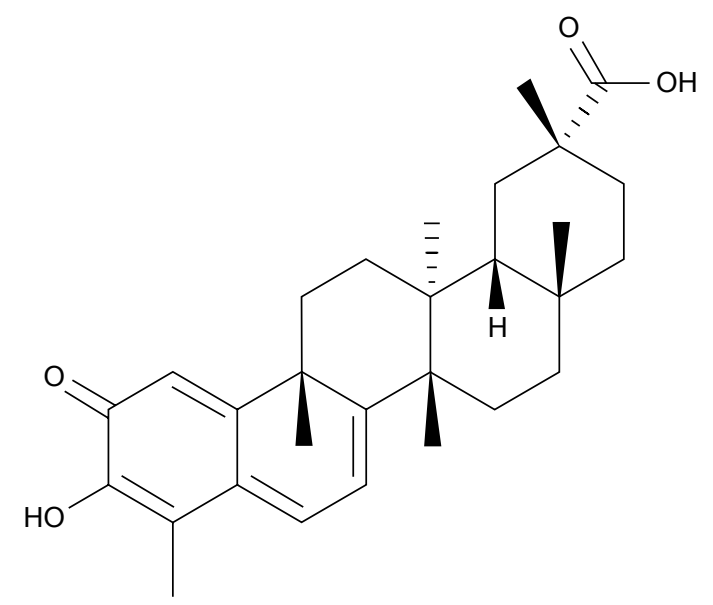

Figure I Chemical structure of celastrol.

activity in androgen-independent prostate cancer both in vitro and in vivo. ${ }^{10}$ Despite its potential in cancer treatment, further therapeutic application of CL is affected by the poor water solubility, low oral bioavailability, and multiple side effects. ${ }^{11,12}$ Furthermore, harmful solvents such as dimethyl sulfoxide (DMSO) need to be used to solubilize the hydrophobic CL, and this limits its clinical use. ${ }^{13,14}$ In spite of this, to overcome these physicochemical and pharmacokinetic deficiencies, and to reduce the amount of the effective dose, the nanoencapsulation of CL can represent a useful strategy. ${ }^{15,16}$

To date, only two studies have explored the effectiveness of nanoencapsulated CL in the treatment of prostate cancer. ${ }^{14,17}$ In the first study, CL-loaded PEGylated liposomes displayed anticancer activity in $\mathrm{VCaP}$ cells, comparable to that of the free drug reconstituted in DMSO.${ }^{14}$ Later, cellpenetrating peptide-coated CL-loaded nanostructured lipid carriers noticeably enhanced antitumor activity in vitro and in vivo in comparison with free tripterine. ${ }^{17}$ However, the $\mathrm{CL}$ encapsulation in clinically suitable polymeric nanosystems has not yet been investigated.

In our previous studies, the hydrophobic polyester poly-( $\varepsilon$-caprolactone) (PCL), as block copolymer or in combination with many biocompatible/biodegradable polymers, was successfully proposed for the formulation of nanoparticles (NPs) in the prevention ${ }^{18}$ and treatment ${ }^{19}$ of prostate cancer, as well as for the controlled release of white tea extract. ${ }^{20}$

The present work is aimed at investigating the encapsulation of CL in novel polymeric PCL NPs as potential prototypes for prostate cancer treatment. The NPs were prepared by a nanoprecipitation method, and characterized in terms of morphology, encapsulation efficiency, chemical properties, and in vitro release kinetics. Moreover, the cellular uptake was visualized by confocal laser scanning microscopy, and antiproliferative efficacy of NPs was evaluated by in vitro cytotoxicity assays toward a panel of hormone-sensitive and hormone-independent human prostate carcinoma cells. In order to elucidate if nanoencapsulation can modify/retain the mechanistic identity of free compound, Western blot analyses were assessed to test the efficacy of the treatments on apoptosis, migration, proliferation, and angiogenesis in human prostate cancer cells.

\section{Materials and methods Chemicals and reagents}

CL was purchased from Baoji Guokang Bio-Technology Co., Ltd, (Shaan'xi Province, People's Republic of China). PCL and Pluronic ${ }^{\circledR}$ F-127 were purchased from Sigma-Aldrich Co. (St Louis, MO, USA). All solvents and other chemicals were obtained from Sigma-Aldrich Co., were analytical grade and were used without further purification. Primary antibodies were obtained from Cell Signaling Technology (Beverly, MA, USA) or Santa Cruz Biotechnology Inc. (Dallas, TX, USA). Anti-mouse and anti-rabbit secondary antibody horseradish peroxidase conjugates were obtained from Cell Signaling Technology. BCA (bicinchoninic acid) protein assay kit was obtained from Pierce (Rockford, IL, USA). Novex precast Tris-glycine gels were purchased from Bio-Rad Laboratories Inc. (Hercules, CA, USA).

\section{Formulation of NPs}

CL-loaded NPs were prepared by a nanoprecipitation method dissolving PCL (47.5 mg) and CL (2.5 mg) in $3 \mathrm{~mL}$ of acetonitrile, and adding the solution dropwise to $20 \mathrm{~mL}$ of Pluronic F-127 solution $(1 \%, w / w)$ under gentle magnetic stirring. The resulting NPs' suspension was evaporated under stirring $(265.5 \times g)$ at room temperature to remove the organic solvent. NPs were centrifuged at $2655 \times g$ for 5 minutes and washed three times with water to remove the unencapsulated CL. Unloaded NPs were produced in a similar manner and used as comparison. Additionally, fluorescent fluorescein isothiocyanate-loaded NPs were prepared by adding $1.0 \mathrm{mg}$ of FITC instead of CL, and used for the cellular uptake experiments.

\section{Particle size and polydispersity index (PDI)}

Mean diameter, size distribution, and PDI of the NPs were measured by photon correlation spectroscopy with Nanosizer S90 particle size analyzer (Malvern Instruments, Malvern, UK). The samples were diluted with deionized water and analyzed at $25^{\circ} \mathrm{C}$. Each analysis was carried out in triplicate. 


\section{Zeta potential}

Zeta potential of the NPs was measured at $25^{\circ} \mathrm{C}$ with a Zeta Plus analyzer (Brookhaven Instruments, Holtsville, NY, USA). The samples were diluted with distilled water and sonicated for several minutes before measurement. Data were obtained by the averaging of three measurements.

\section{Scanning electron microscopy (SEM) analysis}

Morphological examination of NPs was performed by SEM (model DSM 962; Carl Zeiss Meditec AG, Jena, Germany). A drop of NPs aqueous suspension was placed on aluminum stub and dried under vacuum for 12 hours. The samples were then analyzed at $20 \mathrm{kV}$ acceleration voltage after gold sputtering, under an argon atmosphere.

\section{Fourier transform infrared spectroscopy (FTIR)}

The chemical composition of the CL raw material, unloaded and CL-loaded NPs was analyzed by FTIR spectral measurements by using a Nicolet Nexus FTIR spectrophotometer at a resolution of $4 \mathrm{~cm}^{-1}$ in $\mathrm{KBr}$ pellets, in the range $400-4,000 \mathrm{~cm}^{-1}$.

\section{Raman spectroscopy}

The chemical composition of the CL raw material, empty NPs, and CL-loaded NPs was analyzed by Raman spectroscopy (Senterra Raman microscope; Bruker Optik GmbH, Ettlingen, Germany) with a laser excitation of $532 \mathrm{~nm}$ and $5 \mathrm{~mW}$ nominal power and a $50 \times$ objective. The spectra were recorded in the $70-4,500 \mathrm{~cm}^{-1}$ range, with a resolution of $9 \mathrm{~cm}^{-1}$, an integration time of 3 seconds and 6 co-additions.

\section{Thermal analyses}

The thermal properties of the empty and CL-loaded NPs were determined using a differential scanning calorimeter (DSC) Q100 V 9.0 (TA Instruments, New Castle, DE, USA). Indium was used to calibrate the instrument. The thermograms of samples were obtained at a scanning rate of $20^{\circ} \mathrm{C} / \mathrm{min}$ in $20^{\circ} \mathrm{C}-350^{\circ} \mathrm{C}$ temperature range and performed under an $\mathrm{AR}$ purge $(50 \mathrm{~mL} / \mathrm{min})$. The thermal measurements were carried out on pure CL, freeze dried unloaded and CL-loaded NPs.

\section{Drug loading content (DLC), encapsulation efficiency, and yield of production}

To determine the drug loading, an aliquot of NPs (1 mg) was dissolved in $1.0 \mathrm{~mL}$ of acetonitrile. The solution was filtered through a $0.2 \mu \mathrm{m}$ syringe filter and analyzed by ultraviolet-visible spectroscopy (Cary 3, Varian) at $422 \mathrm{~nm}$. The amount of CL was calculated by referring to the calibration curve in acetonitrile (standard solutions in the range of $\left.1.0-30 \mu \mathrm{g} / \mathrm{mL} ; \mathrm{y}=0.0341 \times-0.0009 ; R^{2}=0.9999\right)$.

The DLC\%, drug entrapment efficiency (EE\%), and yield of production (YP \%) of NPs were presented by the following equations, respectively:

$$
\begin{gathered}
\text { DLC } \%=\frac{\text { Weight of drug in NPs }}{\text { Weight of NPs }} \times 100 \% \\
\mathrm{EE} \%=\frac{\text { Actual CL content }}{\text { Theoretical CL content }} \times 100 \% \\
\mathrm{YP} \%=\frac{\text { Weight of NPs recovered }}{\text { Weight of polymer and CL fed initially }} \times 100 \%
\end{gathered}
$$

\section{In vitro release studies}

Approximately $2.0 \mathrm{mg}$ of CL-loaded NPs and pure CL were placed into dialysis bags and suspended in $20 \mathrm{~mL}$ of phosphate-buffered saline (PBS), $\mathrm{pH} 7.4$, then incubated at $37^{\circ} \mathrm{C}$ and stirred at $106.2 \times \mathrm{g}$. At set time points, $1.0 \mathrm{~mL}$ of solution was withdrawn and replaced with fresh solution. Samples were filtered and CL concentration was assayed spectrophotometrically at $422 \mathrm{~nm}$, thus calculated by referring to the respective calibration curve with standard solutions (PBS: ethanol 4:1, v/v) in the range of $1-50 \mu \mathrm{g} / \mathrm{mL}$ $\left(\mathrm{y}=0.0339 \times-0.0091 ; R^{2}=0.9999\right)$.

\section{Cell culture}

The prostate carcinoma LNCaP, DU-145, and PC3 cells were obtained from American Type Culture Collection (Manassas, VA, USA). The cells were cultured in Roswell Park Memorial Institute 1640 and were maintained under standard cell culture conditions supplemented with $10 \%$ FBS and $1 \%$ penicillin/ streptomycin at $37^{\circ} \mathrm{C}$ and $5 \% \mathrm{CO}_{2}$ environment.

\section{Cell growth and viability assay}

The cell growth and viability were assessed by the 3-[4,5dimethylthiazol-2-yl]-2,5-diphenyl tetrazolium bromide (MTT) assay as previously described. ${ }^{21}$ Briefly, the cells were plated at $1 \times 10^{4}$ per well in $200 \mu \mathrm{L}$ of complete culture medium. The next day, cells were treated with native CL or CL-loaded NPs for 24 hours, at $0.5,1.0$, and $2.0 \mu \mathrm{M}$ equivalent CL concentrations. Each concentration was repeated in ten wells. After incubation for the specified time at $37^{\circ} \mathrm{C}$ in a humidified incubator, cell viability was determined. 
MTT (5 mg/mL in PBS) was added to each well and incubated for 2 hours, after which the plate was centrifuged at $500 \times g$ for 5 minutes at $4{ }^{\circ} \mathrm{C}$.

The MTT solution was aspirated from the wells using vacuum, and $0.2 \mathrm{~mL}$ of buffered DMSO was added to each well. After a 10-minute mixing, the absorbance was recorded on a microplate reader at the wavelength of $540 \mathrm{~nm}$. The effect of each agent on growth inhibition was assessed as percentage of cell viability in which vehicle-treated cells were taken as $100 \%$ viable. The experiment was repeated three times with similar outcomes.

\section{Cellular uptake}

Fluorescence microscopy was utilized to examine the cellular uptake of the CL-NPs. Cells were plated in a two-chambered slide at 5,000 cells/well and allowed to grow for 18 hours. Post-attachment, the cells were incubated for 120 minutes with FITC-loaded NPs. Unbound micelles were removed by washing three times with PBS, the cells were mounted using Gold Antifade Reagent containing 4',6-diamidino2-phenylindole (Thermo Fisher Scientific, Waltham, MA, USA), and the mountant was allowed to cure overnight in the dark at room temperature. The images were examined under Nikon Eclipse Ti inverted microscope (Nikon Instruments, Melville, NY, USA), and then captured with a camera attached to the microscope. The experiment was repeated four times with similar results.

\section{Protein extraction, quantification, and Western blotting}

Prostate cancer PC3 cell lines were harvested after various treatments and whole cell lysates were prepared, resolved by sodium dodecyl sulfate denaturing and reducing polyacrylamide gel electrophoresis followed by Western blot analysis as described previously. ${ }^{22}$ Briefly, cells were washed with cold $1 \times$ PBS (10 mM, pH 7.4), incubated in ice-cold RIPA (radioimmunoprecipitation assay) lysis buffer freshly supplemented with $\mathrm{Na}_{3} \mathrm{VO}_{4}(100 \mathrm{mM})$, phenylmethanesulfonyl fluoride (1 mM) and protease inhibitor cocktail Set III (Calbiochem, La Jolla, CA, USA) on ice for 15 minutes. Cells were scraped into a microfuge tube, disaggregated through a $22 \mathrm{G}$ needle, and lysates were cleared by centrifugation at $14,000 \times g$ for 25 minutes at $4^{\circ} \mathrm{C}$, and the supernatants (lysate) were stored at $-80^{\circ} \mathrm{C}$ until used. The concentration of the protein in lysates was determined by the BCA protein assay kit as per the manufacturer's protocol (Thermo Fisher Scientific), and Western blot analysis was performed as described elsewhere. ${ }^{23}$ Equal quantities of protein 30-40 $\mu \mathrm{g}$ were electrophoresed on polyacrylamide gel electrophoresis, transferred onto nitrocellulose membranes, blocked with 5\% non-fat dry milk in phosphatebuffered saline Tween 20 , followed by overnight incubation at $4^{\circ} \mathrm{C}$, with respective primary antibodies. After several washes, blots were incubated for 1 hour at room temperature with appropriate horse radish peroxidase-conjugated secondary antibodies, and binding was visualized by chemiluminescent detection system using enhanced chemiluminescence SuperSignal West Pico kit, and developed by the BioRad ultraviolet-trans camera or on film. Each blot was stripped and re-probed with $\beta$-actin as housekeeping assessing equal protein loading, and data are presented as the relative density of protein bands normalized to $\beta$-actin.

\section{Cell migration}

Cell migration was performed by using Radius ${ }^{\mathrm{TM}} 24-\mathrm{Well}$ Cell Migration Assay kit from Cell Biolabs Inc. (San Diego, CA, USA) by following manufacturer's instructions.

\section{Statistical analysis}

All data were subjected to one-way analysis of variance (GraphPadPrism, version 5.03). Individual differences were evaluated using a nonparametric post hoc test (Tukey's test) and considered statistically significant at $P<0.05$.

\section{Results and discussion}

Nanotechnology-based strategies are an emerging and promising approach with a great potential to facilitate the chemoprevention and treatment of prostate cancer. ${ }^{24-32}$ Meanwhile, the natural products are valuable sources of bioactive compounds that have drawn a great deal of attention because of their ability to suppress cancers as well as to reduce the risk of cancer development. ${ }^{33-39}$ Potential advantages of NP-based therapeutic products are the capability to revert unfavorable physicochemical properties of bioactive molecules (including phytochemicals/natural products) to desirable pharmacokinetic profiles, improve delivery of therapeutics across biological barriers and compartments, reduce intrinsic cytotoxicity, control release, and enhance therapeutic efficacy by selective delivery of therapeutics to biological targets. ${ }^{40-42}$ In this context, the good pharmacological activity and the physicochemical limitations such as low solubility, low permeability, and poor bioavailability, therefore make CL a suitable candidate for nanoencapsulation. ${ }^{15,43}$

\section{Formulation of NPs}

PCL-based NPs were prepared by the nanoprecipitation method and investigated with the goal to enhance its dispersity in aqueous solution and the resulting bioavailability. 


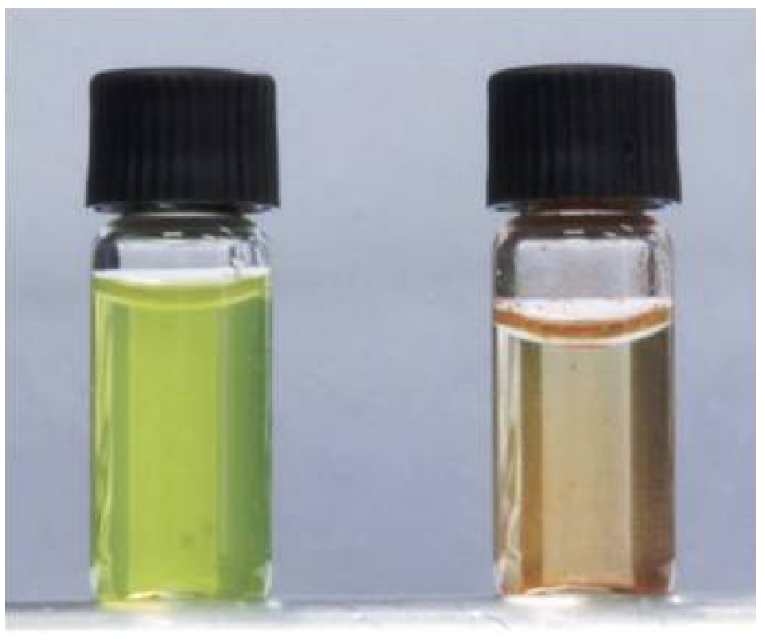

Figure 2 Photograph of CL-loaded NPs (left), and CL raw material (right) after dispersion in water.

Abbreviations: $\mathrm{CL}$, celastrol; NPs, nanoparticles.

As illustrated in Figure 2, the CL-loaded NPs in water provided a clear and fully dispersible formulation, with CL's natural color intense yellow. In contrast, free CL is not dissolved in aqueous media with the visible precipitation of red crystals in the solution. This suggests that CL-NPs could represent a valid alternative to the use of toxic solubilizing agents, such as DMSO, normally required for the administration of CL.

\section{Characterization of NPs}

The prepared NPs showed an average diameter lower than $200 \mathrm{~nm}(189.1 \pm 2.9 \mathrm{~nm}$ and $175.5 \pm 4.7 \mathrm{~nm}$ for unloaded and CL-loaded NPs, respectively). Besides, as depicted in Figure 3, both NP formulations are characterized by a unimodal particle size distribution, as also confirmed by the obtained low PDIs $(<0.2)$, typical of monodispersed systems (Table 1). Moreover, the zeta potential of the uncoated NPs was negative $(-15.6 \pm 0.3 \mathrm{mV})$, as expected, without significant differences between the loaded batch $(-16.2 \pm 0.4 \mathrm{mV})$, thus reflecting a steric stabilization effect that avoids aggregation of the fine particles in the colloidal system.

SEM photomicrographs revealed similar morphological aspects for both kinds of NPs (loaded and unloaded), with spherical shape and without a tendency to aggregate (Figure 3), suggesting that loading of CL into NPs did not significantly affect their size and morphology.

As far as the drug EE is concerned, the PCL demonstrated a good encapsulation capability of the CL into the polymeric matrix, with $\mathrm{EE} \%$ values of $65.2 \% \pm 1.1 \%$, corresponding to a DLC value of $3.26 \% \pm 0.1 \%$ (Table 1). This finding can be ascribed to the high affinity between the hydrophobic polymer and CL molecules. Moreover, YPs obtained were in the range of $50 \%$.

Figure 4 shows the comparison of FTIR spectra for pure CL, unloaded and CL-loaded NPs. The CL spectrum showed a band $\sim 2,950-2,850 \mathrm{~cm}^{-1}$ corresponding to $-\mathrm{OH}$ stretching, a strong characteristic peak at $1,702 \mathrm{~cm}^{-1}$ attributed to $-\mathrm{C}=\mathrm{O}$ stretching, the band at $1,582-1,510 \mathrm{~cm}^{-1}$ due to $\mathrm{C}-\mathrm{C}$ stretch in ring, a peak centered at $1,440 \mathrm{~cm}^{-1}$ assigned to $\mathrm{C}-\mathrm{H}$ bending, and the band at 1,323-1,110 $\mathrm{cm}^{-1}$ corresponding to $\mathrm{C}-\mathrm{O}$ stretch. ${ }^{18}$

According to Elzein et $\mathrm{al}^{44}$ unloaded NPs shared the characteristic peaks of PCL, with asymmetric and symmetric stretching of $\mathrm{CH}_{2}$ at 2,949 and 2,865 $\mathrm{cm}^{-1}$, respectively. Moreover, a specific signal for the carbonyl-stretching mode is indicated at $1,725 \mathrm{~cm}^{-1}$, whereas the $\mathrm{C}-\mathrm{O}$ and $\mathrm{C}-\mathrm{C}$ stretching at $1,293 \mathrm{~cm}^{-1}$, asymmetric COC stretching at $1,240 \mathrm{~cm}^{-1}$, and $\mathrm{OC}-\mathrm{O}$ stretching at $1,190 \mathrm{~cm}^{-1}$. On the other hand, the presence of new absorption band at $1,660-1,500 \mathrm{~cm}^{-1}$ in the CL-loaded NPs' spectrum supported the successful encapsulation of CL into nanosystems. Additionally, we observed two shifts for the carbonyl stretching to $1,727 \mathrm{~cm}^{-1}$ and for $\mathrm{OC}-\mathrm{O}$

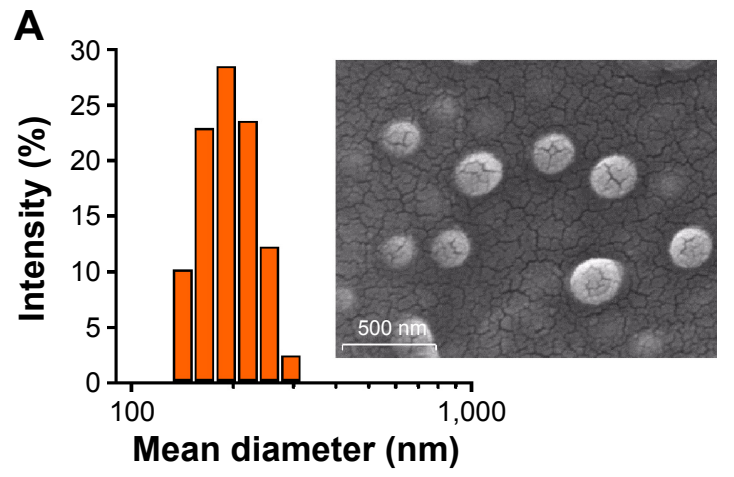

Figure 3 Particle size distributions and SEM images.

Note: (A) Empty and (B) CL-loaded NPs.

Abbreviations: CL, celastrol; NPs, nanoparticles; SEM, scanning electron microscopy.

B

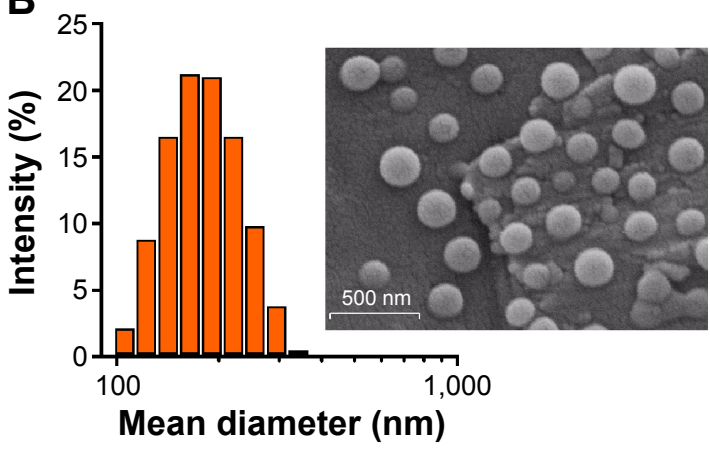


Table I Particle size (PS), polydispersity index (PDI), zeta potential (ZP), entrapment efficiencies (EE), drug loading content (DLC), and yields of production (YPs) of formulated NPs

\begin{tabular}{lllllll}
\hline Batch & PS $(\mathbf{n m})$ & PDI & ZP $(\mathbf{m V})$ & \%EE & \%DLC & \%YP \\
\hline Unloaded NPs & $189.1 \pm 2.9$ & $0.064 \pm 0.02$ & $-15.6 \pm 0.3$ & - & - & $49.4 \pm 2.2$ \\
CL-loaded NPs & $175.5 \pm 4.7$ & $0.138 \pm 0.07$ & $-16.2 \pm 0.4$ & $65.2 \pm 1.1$ & $3.26 \pm 0.05$ & $51.3 \pm 1.9$ \\
\hline
\end{tabular}

Note: Data are expressed as mean \pm standard deviation $(n=3)$

Abbreviations: CL, celastrol; NPs, nanoparticles.

stretching to $1,186 \mathrm{~cm}^{-1}$, which might be explained by a weak interaction between the $\mathrm{CL}$ and the polymeric chains.

Results obtained through Raman studies (Figure 5) were in good agreement with FTIR analyses. The spectrum of unloaded NPs, containing only PCL, showed strong bands at $3,000-2,800 \mathrm{~cm}^{-1}$ and bands between 1,400 and $1,500 \mathrm{~cm}^{-1}$, which correspond to asymmetric and symmetric $\mathrm{CH}_{2}$ stretching, respectively. The peak centered at $1,725 \mathrm{~cm}^{-1}$ was characteristic of carbonyl stretching, and band at $1,293 \mathrm{~cm}^{-1}$ was attributed to $\mathrm{C}-\mathrm{O}$ and $\mathrm{C}-\mathrm{C}$ stretching vibrations. ${ }^{45,46}$

In CL spectrum, the bands at $1,650-1,500 \mathrm{~cm}^{-1}$ are due to the $\mathrm{C}=\mathrm{C}$ stretching vibrations, and the intense absorption peak at $1,445 \mathrm{~cm}^{-1}$, related to the axial deformation of $\mathrm{C}=\mathrm{O}$ of carboxylic acid, is also consistent with the $\mathrm{OH}$ deformation in carboxylic acid. Moreover, the peak registered at $1,385 \mathrm{~cm}^{-1}$ is attributed to $\mathrm{C}-\mathrm{H}$ symmetric deformation of $\mathrm{CH}_{3}$. The overlapping signals corresponding to the PCL and $\mathrm{CL}$, detected in CL-loaded NPs, confirmed the presence of encapsulated CL.

Additionally, DSC experiments were performed to investigate the physical state of the CL encapsulated in the NPs, as well as CL and polymer interactions. The DSC thermogram of CL (Figure 6) shows a sharp endothermic

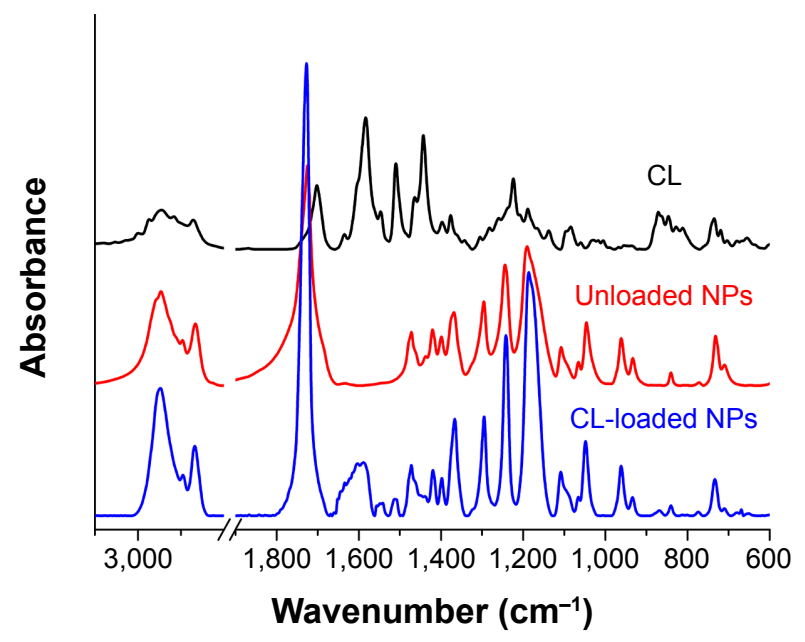

Figure 4 FTIR spectra of CL raw material, unloaded NPs, and CL-loaded NPs. Abbreviations: FTIR, Fourier transform infrared spectroscopy; CL, celastrol; NPs, nanoparticles. peak at $156.2^{\circ} \mathrm{C}$, which corresponds to the melting point of $\mathrm{CL}$, and an exothermic peak at $210.1^{\circ} \mathrm{C}$, probably due to the degradation of CL. ${ }^{47}$

The DSC scan of unloaded NPs exhibits the characteristic melting endotherm of semi-crystalline PCL at $64.55^{\circ} \mathrm{C}$, shifted to $59.66^{\circ} \mathrm{C}$ in CL-loaded NPs thermogram, suggesting intermolecular interactions between the polymer and CL. ${ }^{18}$

Furthermore, the disappearance of the endothermic peak observed in the DSC curve of CL-loaded NPs indicates that CL was dispersed within NPs in an amorphous state, or that it is present as a solid molecular dispersion in the polymer matrix.

\section{In vitro release studies}

The highly hydrophobic nature of the $\mathrm{CL}$ is one of the major limiting factors for its clinical development. In order to avoid the use of toxic organic solvents, as well as to provide controlled release and protection, the nanoformulation is demonstrating to be a useful strategy. ${ }^{16}$ Figure 7 shows the release profiles of free CL and CL encapsulated in PBS, at $\mathrm{pH}$ 7.4. As can be noted, the amount of CL raw material dissolved in the media results are negligible (maximum 6\%, after 72 hours), due to the poor solubility of the molecule. Conversely, the CL released from NPs result significantly increased, with percentages of native compound recovered of

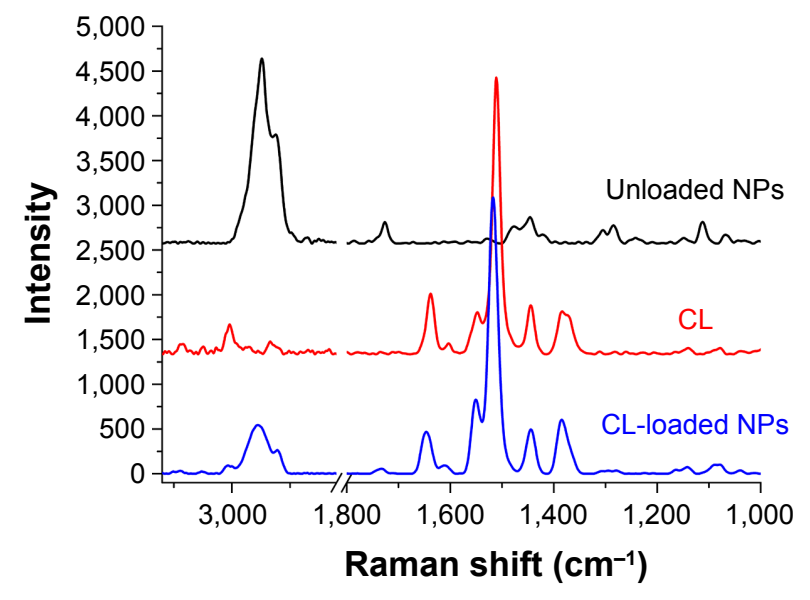

Figure 5 Raman spectra of CL raw material, unloaded NPs, and CL-loaded NPs. Abbreviations: $\mathrm{CL}$, celastrol; NPs, nanoparticles. 


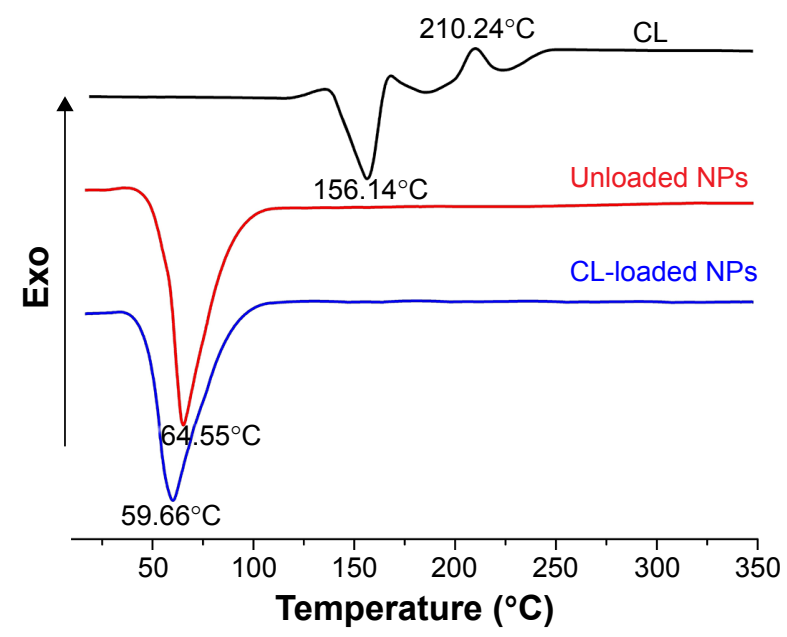

Figure 6 DSC curves of CL raw material, unloaded NPs, and CL-loaded NPs. Abbreviations: DSC, differential scanning calorimeter; CL, celastrol; NPs, nanoparticles; Exo, exothermic.

$17 \%, 27 \%$, and $60 \%$ after 24,48 , and 72 hours, respectively. The obtained results demonstrated a more favorable increase of CL solubility after the encapsulation into polymeric NPs than that of liposome formulations previously reported. ${ }^{14}$

\section{Cytotoxicity and cellular uptake of NPs}

In vitro cytotoxic activity of free CL, CL-NPs and emptyNPs was evaluated by MTT assay using androgen-responsive (LNCaP) and androgen-unresponsive (DU-145 and PC3) prostate cancer cells. More specifically, cells were incubated in presence of increasing CL-NPs concentrations (equivalent CL concentrations $0.5,1.0$, and $2.0 \mu \mathrm{M}$ were run in parallel) for 24 hours.

On the whole, the results demonstrated that CL encapsulated in NPs was more effective against both cancer cell lines than free CL, in a dose-dependent manner (Figure 8). However, differences in sensitivity toward both CL and

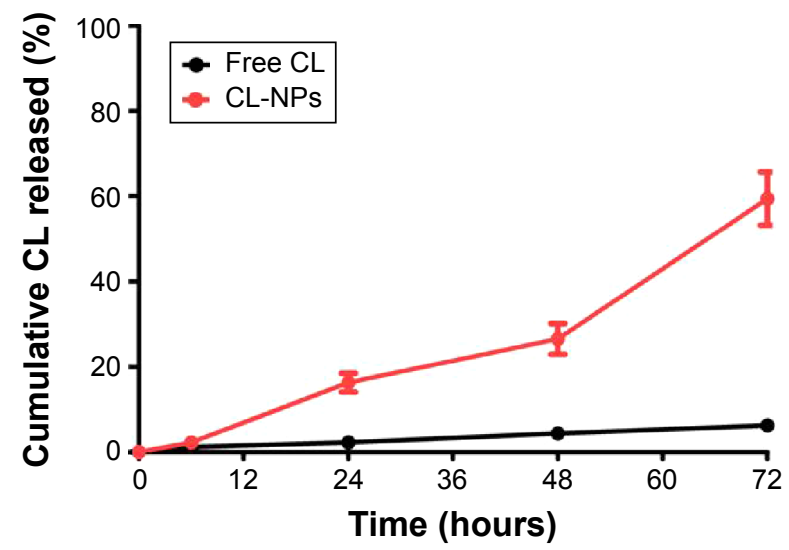

Figure 7 In vitro release profiles of free CL and CL-NPs in PBS ( $\mathrm{pH} 7.4$ ), performed for 72 hours.

Abbreviations: CL, celastrol; NPs, nanoparticles; PBS, phosphate-buffered saline.
CL-NPs exposure were found for the tested tumor cells. In particular, in LNCaP cells no influence on viability inhibition was observed after treatment with both free CL and CL-NPs at $0.5 \mu \mathrm{M}$ concentration, with respect to untreated cells (control) (Figure 8A). The increase of free-CL dose to 1.0 and $2.0 \mu \mathrm{M}$ concentrations improved the anti-proliferative activity with an inhibition of cell viability by $43 \%$ and $82 \%$, respectively ([inhibitory concentration] $\mathrm{IC}_{50}$ dose of $1.32 \mu \mathrm{M}$ ). Treatment with NPs at comparable doses of CL-NPs resulted in an enhanced reduction of cell viability $(42 \%)$ at $1.0 \mu \mathrm{M}$ concentration while a concomitant inhibition of approximately $80 \%$ was observed at $2.0 \mu \mathrm{M}$.

Similar experiments were performed on DU-145 cells where native agent resulted in a cell viability decrease of $10 \%, 74 \%$, and $88 \%$ respectively at $0.5,1.0$, and $2.0 \mu \mathrm{M}$ concentrations, with an $\mathrm{IC}_{50}$ of $0.82 \mu \mathrm{M}$. CL-NPs also demonstrated a significant increase in cytotoxicity activity with 1 and $2 \mu \mathrm{M}$, demonstrating $62 \%$, and $86 \%$ inhibition of cell viability, respectively, which was comparable to the native agent (Figure 8B).

Finally, at the same doses, CL-NPs showed an enhanced growth inhibition of PC3 cells of $64 \%$ and $80 \%$, respectively, thus resulting in a significant cell viability reduction of approximately $40 \%$, and $32 \%$, compared with that of free $\mathrm{CL}\left(\mathrm{IC}_{50}\right.$ dose of $\left.1.10 \mu \mathrm{M}\right)$ (Figure 8C).

Similarly to LNCaP, in both DU-145 and PC3 cell lines, no significant differences were observed after treatment at $2.0 \mu \mathrm{M}$ dose.

These results suggest that NPs enhanced the CL cytotoxicity at lower doses by consistently decreasing the androgenindependent prostate cancer cell lines' (DU-145 and PC3) proliferation and viability, thus allowing a reduction of the CL amount administered and limiting the adverse effects. ${ }^{10}$ Importantly, no significant influence on cell viability was observed for empty NPs evaluated, considering the same concentration of the polymer as in CL-NPs at $2.0 \mu \mathrm{M}$, confirming the good biocompatibility of PCL polymer.

According to literature reports, the higher cytotoxicity demonstrated by CL formulated into NPs can be ascribed to the combination of different and mutual mechanisms. For example, the NPs were adsorbed on the cell surface, and this interaction generates a concentration gradient that promotes the drug influx into the cell. ${ }^{48}$ Although part of free CL molecules was actively exported out from the cell by P-gp pumps, NPs are taken up by cells through an endocytosis process, thus resulting in a consistent cellular uptake of the entrapped drug, ${ }^{49}$ which enabled them to escape from the effect of P-gp pumps. Moreover, intracellular delivery of CL-NPs 

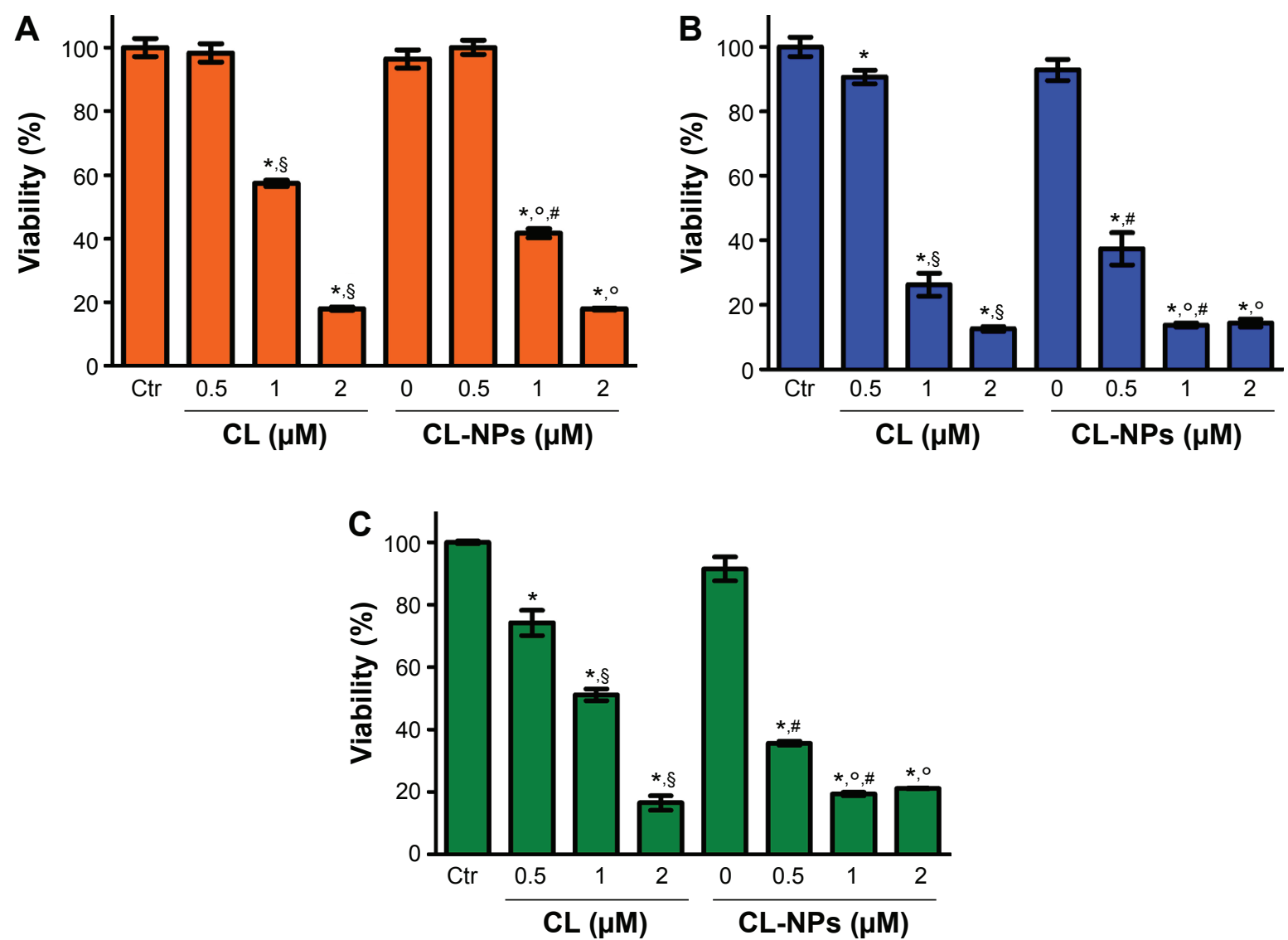

Figure 8 Viability of LNCaP (A), DU-I45 (B), and PC3 (C) cells cultured with empty NPs (CL-NPs 0), and CL-loaded NPs (CL-NPs) for 24 hours, in comparison with that of free $C L$ at the $0.5,1.0$, and $2.0 \mu M$ dose $(n=3)$.

Notes: *Significantly different $(P<0.05)$ from control. ${ }^{\S}$ Significantly different $(P<0.05)$ from other free $C L$ doses. ${ }^{\circ}$ Significantly different $(P<0.05)$ from other $C L-N P s$ doses. "Significantly different $(P<0.05)$ from free $C L$ at equivalent doses.

Abbreviations: $\mathrm{CL}$, celastrol; NPs, nanoparticles; Ctr, control.

leads to a massive drug accumulation and localization near to the site of action, thus allowing for higher impact in terms of efficacy, but retaining the mechanistic identity of free CL. ${ }^{50}$

The cellular uptake of FITC-loaded NPs, in all prostate cancer cell lines, was further investigated by confocal laser scanning microscope. As shown in Figure 9, after 2 hours incubation, NPs encapsulating FITC exhibited higher cellular uptake with strong fluorescence in both the cell cytoplasm and around the nucleus, confirming their internalization and sustained retention by the cancer cells. The cellular uptake efficiency in DU-145 (Figure 9B) and PC3 (Figure 9C) cell lines appeared higher than that of LNCaP (Figure 9A) cells, thus supporting the differences in sensitivity toward CL-NPs exposure observed in cytotoxicity results.

\section{CL-loaded NPs suppress migration of androgen independent prostate cancer cells PC3 compared to pure CL}

As the low/medium concentrations $(0.5 \mu \mathrm{M}$ and $1.0 \mu \mathrm{M})$ CL-NPs showed more effective results than the same concentrations of free CL in PC3 cells, we evaluated the effect of CL in comparison with CL-NPs on cell migration using the lowest (non-toxic) doses $(0.5 \mu \mathrm{M})$ of our nanosystems. PC3 cell migration results, reported in Figure 10, demonstrated that with concentrations of CL that nominally affected proliferation $(0.5 \mu \mathrm{M})$, CL-NPs showed more effective results in preventing cell migration (Figure 10B) than CL treatment at same concentration (Figure 10C). These data suggest that CL-NPs suppress the androgen independent prostate cancer (AIPC) cell migration at sub-cytotoxic concentration, where CL is minimally effective.

\section{CL-loaded NPs modulate apoptosis and suppress proliferation, angiogenesis, and cell cycle protein markers in AIPC (PC3) compared to pure $\mathrm{CL}$}

Apoptosis is a process that comprises the activation of several key players and crucial components of the cell signaling machinery, which trigger inhibition of cell growth and proliferation. CL has demonstrated potential in basic and preclinical studies showing suppression of tumor progression through the modulation of apoptosis, inhibition 

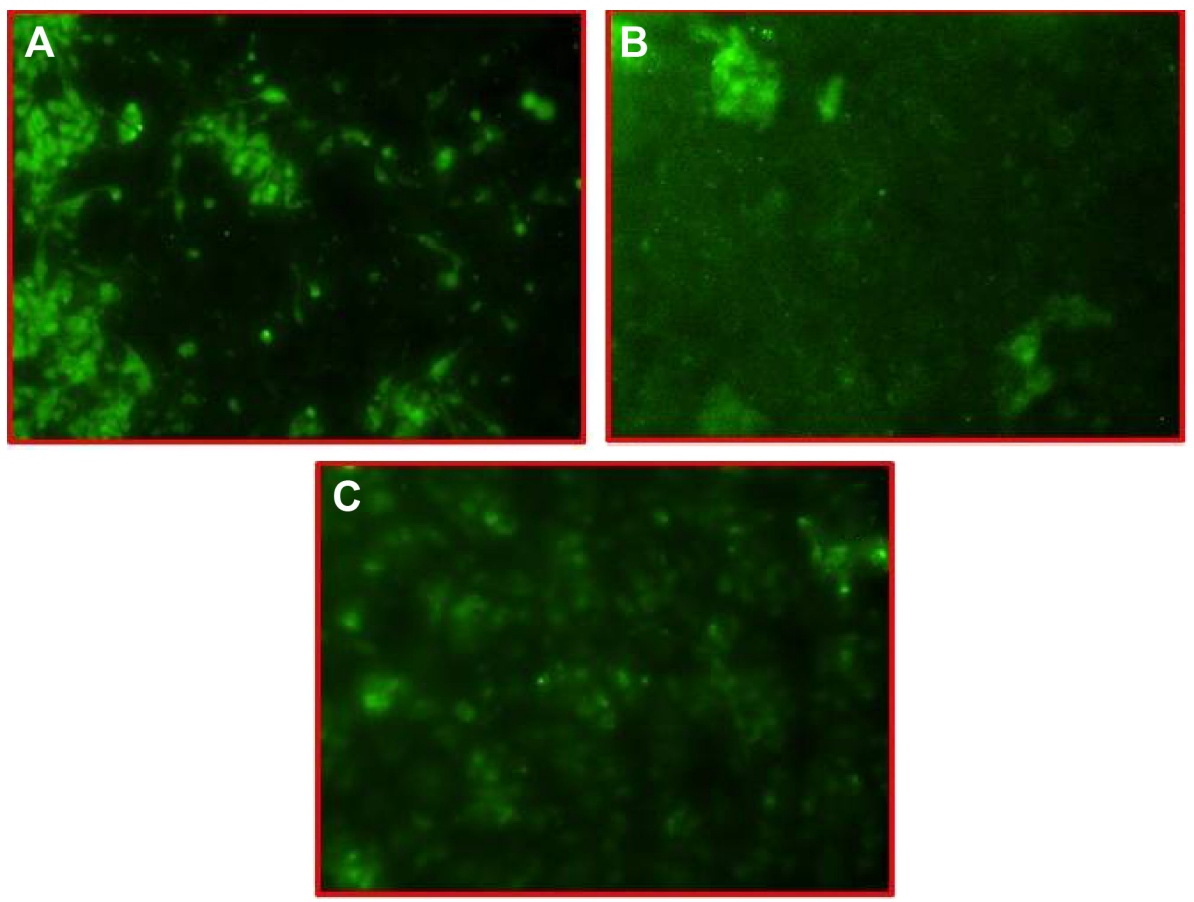

Figure 9 Confocal laser scanning microscopy images.

Notes: (A) LNCaP, (B) DU-145, and (C) PC3 cell lines, after 120 minutes incubation with FITC-loaded NPs at $37.0^{\circ} \mathrm{C}$. The NPs' internalization is observed as green fluorescence of FITC. Magnification 20x.

Abbreviations: NPs, nanoparticles; FITC, fluorescein isothiocyanate.

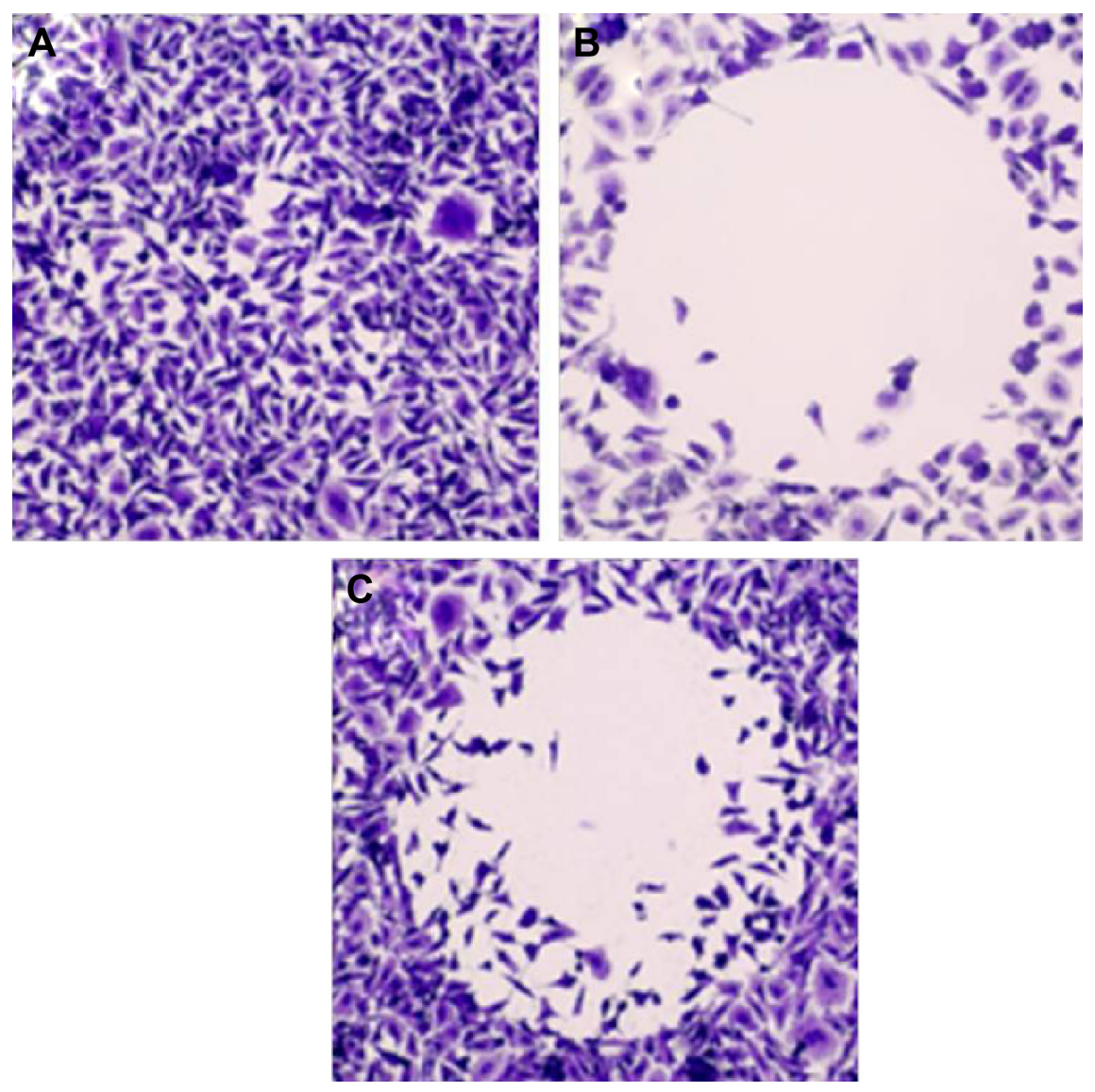

Figure 10 PC3 cell migration results.

Notes: (A) Control, (B) CL-NPs, and (C) free CL, at CL concentration of $0.5 \mu \mathrm{M}$.

Abbreviations: $\mathrm{CL}$, celastrol; NPs, nanoparticles. 
of proliferation, angiogenesis, migration, cell cycle and death receptors of different tumor cell lines. ${ }^{10,51,52}$ Since CL has been shown to induce apoptosis and reduce cell viability, we next investigated the comparative effect of CL and CL-NPs on apoptosis, proliferation, angiogenesis, and cell cycle protein markers, using PC3 as a model cell line. Although CL dose-dependently induced AIPC cells' death, CL-NPs' effect at all instances was more potent than those of nonencapsulated CL. PC3 treated with $0.5,1$, and $2 \mu \mathrm{M}$ of CL-NPs for 24 hours produced significantly greater fold increases in apoptosis compared with CL treated cells, as evident by significant increases in $\mathrm{Bax} / \mathrm{Bcl}-2$ ratio, cleaved PARP
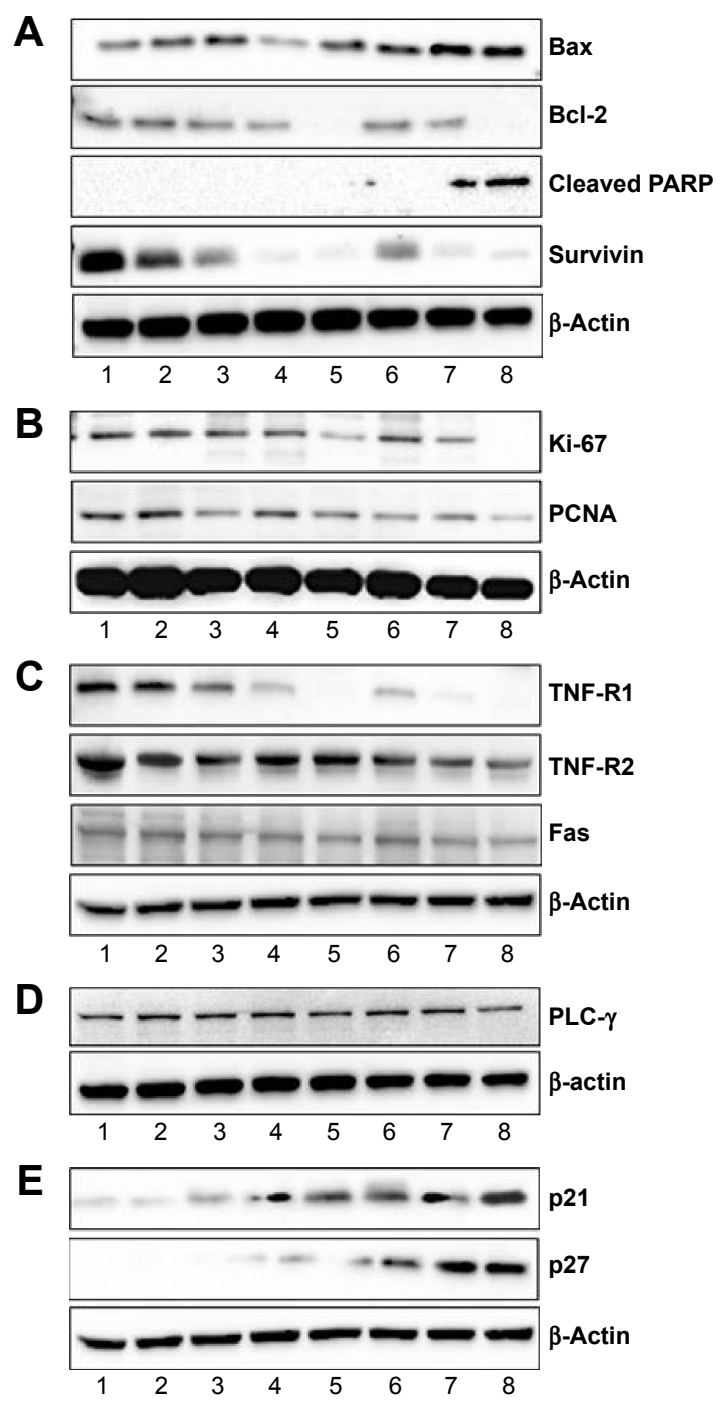

Figure I I Comparative effects of pure CL and CL-NPs at various concentrations on apoptotic, proliferation, death receptor, angiogenesis, and cell cycle biomarkers. Notes: (A) Apoptotic, (B) proliferation, (C) death receptor, (D) angiogenesis, and (E) cell cycle biomarkers. The PC3 cells were treated with each agent and harvested 24 hours after treatments. (Lane I: control; Lane 2: unloaded NPs; Lanes 3-5: pure $\mathrm{CL}$ at $0.5,1.0$, and $2.0 \mu \mathrm{M}$ concentrations, respectively; Lanes 6-8: CL-NPs at 0.5, 1.0, and $2.0 \mu \mathrm{M}$ concentrations, respectively.

Abbreviations: CL, celastrol; NPs, nanoparticles. and decreases in survivin (Figure 11A). PARP is involved in several cellular activities such as regulation of transcription and DNA repair, ${ }^{53}$ and its inhibitors are under intense investigation in various clinical trials. ${ }^{54}$ Similarly, CL-NPs' dose-dependent decreased proliferation (Figure 11B) and death receptors (Figure 11C) was observed by more significant reduction in Ki-67 and PCNA and TNF-R1, TNF-R2 and Fas protein expressions compared to $\mathrm{CL}$ alone. In addition, CL-NPs inhibited angiogenesis, as evoked by stronger PLC- $\gamma$ expression (Figure 11D), and a consistently marked effect on cell cycle arrest, as supported by increases in p 21 and $\mathrm{p} 27$ protein expression (Figure 11E), compared to the expressions induced by only CL treatment.

\section{Conclusion}

Despite the potential suppression of AIPC progression, concerns related to CL bioavailability as well as perceived toxicity associated with high doses and its long-term use affect its clinical development. ${ }^{15,43,55}$ To overcome these obstacles, we have successfully developed the first formulation of CL in novel polymeric PCL NPs. The nanosystems were obtained by a nanoprecipitation technique and extensively characterized in terms of morphology, encapsulation efficiency, chemical properties, and in vitro release kinetics. The CL-NPs exhibited remarkable antiproliferative activities against all cancer cell lines compared to free CL, in a dose-dependent manner, eliciting a major sensitivity on the AIPC cell lines (DU-145 and PC3), with respect to free CL. Mechanistically, our results also showed that CL-NPs trigger a stronger cleavage of PARP, as well as induction of Bax inhibition of Bcl-2, compared to free CL and control. Moreover, a significant decrease in the expression of Ki-67, PCNA (cell proliferation markers), TNF-R1/2 and Fas (death receptor markers), PLC- $\gamma$ (marker of angiogenesis), as well as induction of p21 and p27 (markers of cell cycle arrest) in lysates of PC3 cells treated with CL-NPs compared with free $\mathrm{CL}$ and controls, have been observed. Although these experiments have been performed in a two-dimensional culture setting, the results consistently indicate a potential of these novel polymeric nanotechnology-based PCL NPs as promising carrier to make bioavailable $\mathrm{CL}$, providing a new opportunity in the treatment of prostate cancer.

\section{Acknowledgments}

The authors gratefully acknowledge the Regione Autonoma della Sardegna for financial support of grant CRP 25920, awarded to MS within the frame of "Legge regionale n. 7/2007, promozione della ricerca scientifica 
e dell'innovazione tecnologica in Sardegna, - Annualità 2010", and the Mentored Research Scholar Grant (MRSG11-019-01-CNE), awarded to IAS from the American Cancer Society (USA). The authors also thank Rahime Jashari for help with Western blot analysis, and Dr Davide Carboni for help with DSC analysis.

\section{Disclosure}

The authors report no conflicts of interest in this work.

\section{References}

1. Venkatesha SH, Astry B, Nanjundaiah SM, Yu H, Moudgil KD. Suppression of autoimmune arthritis by Celastrus-derived Celastrol through modulation of pro-inflammatory chemokines. Bioorg Med Chem. 2012; 20(17):5229-5234.

2. Kim DY, Park JW, Jeoung D, Ro JY. Celastrol suppresses allergeninduced airway inflammation in a mouse allergic asthma model. Eur J Pharmacol. 2009;612(1-3):98-105.

3. Nanjundaiah SM, Venkatesha SH, Yu H, Tong L, Stains JP, Moudgil KD. Celastrus and its bioactive celastrol protect against bone damage in autoimmune arthritis by modulating osteoimmune cross-talk J Biol Chem. 2012;287(26):22216-22226.

4. Neef DW, Jaeger AM, Thiele DJ. Heat shock transcription factor 1 as a therapeutic target in neurodegenerative diseases. Nat Rev Drug Discov. 2011;10(12):930-944.

5. LuZ, Jin Y, Qiu L, Lai Y, Pan J. Celastrol, a novel HSP90 inhibitor, depletes Bcr-Abl and induces apoptosis in imatinib-resistant chronic myelogenous leukemia cells harboring T315I mutation. Cancer Lett. 2010; 290(2):182-191.

6. Huang Y, Zhou Y, Fan Y, Zhou D. Celastrol inhibits the growth of human glioma xenografts in nude mice through suppressing VEGFR expression. Cancer Lett. 2008;264(1):101-106.

7. Jang SY, Jang SW, Ko J. Celastrol inhibits the growth of estrogen positive human breast cancer cells through modulation of estrogen receptor alpha. Cancer Lett. 2011;300(1):57-65.

8. Kim Y, Kang H, Jang SW, Ko J. Celastrol inhibits breast cancer cell invasion via suppression of NF-kB-mediated matrix metalloproteinase-9 expression. Cell Physiol Biochem. 2011;28(2):175-184.

9. Yang H, Chen D, Cui QC, Yuan X, Dou QP. Celastrol, a triterpene extracted from the chinese "Thunder of god vine," is a potent proteasome inhibitor and suppresses human prostate cancer growth in nude mice. Cancer Res. 2006;66(9):4758-4765.

10. Dai Y, Desano J, Tang W, et al. Natural proteasome inhibitor celastrol suppresses androgen-independent prostate cancer progression by modulating apoptotic proteins and NF-kappaB. PLoS One. 2010;5(12): e14153.

11. Qi X, Qin J, Ma N, Chou X, Wu Z. Solid self-microemulsifying dispersible tablets of celastrol: formulation development, characterization and bioavailability evaluation. Int J Pharm. 2014;472(1-2):40-47.

12. Liu Z, Ma L, Zhou GB. The main anticancer bullets of the Chinese medicinal herb, thunder god vine. Molecules. 2011;16(6):5283-5297.

13. Salminen A, Lehtonen M, Paimela T, Kaarniranta K. Celastrol: molecular targets of Thunder God Vine. Biochem Biophys Res Commun. 2010;394(3):439-442.

14. Wolfram J, Suri K, Huang Y, et al. Evaluation of anticancer activity of celastrol liposomes in prostate cancer cells. J Microencapsul. 2014; 31(5):501-507.

15. Song J, Shi F, Zhang Z, et al. Formulation and evaluation of celastrolloaded liposomes. Molecules. 2011;16(9):7880-7892.

16. Chen Y, Zhou L, Yuan L, Zhang ZH, Liu X, Wu Q. Formulation, characterization, and evaluation of in vitro skin permeation and in vivo pharmacodynamics of surface-charged tripterine-loaded nanostructured lipid carriers. Int J Nanomedicine. 2012;7:3023-3032.
17. Yuan L, Liu C, Chen Y, Zhang Z, Zhou L, Qu D. Antitumor activity of tripterine via cell-penetrating peptide-coated nanostructured lipid carriers in a prostate cancer model. Int J Nanomedicine. 2013;8: 4339-4350.

18. Sanna V, Siddiqui IA, Sechi M, Mukhtar H. Resveratrol-loaded nanoparticles based on poly(epsilon-caprolactone) and poly(D,L-lactic-coglycolic acid)-poly(ethylene glycol) blend for prostate cancer treatment. Mol Pharm. 2013;10(10):3871-3881.

19. Sanna V, Roggio AM, Posadino AM, et al. Novel docetaxel-loaded nanoparticles based on Poly(lactide-co-caprolactone) and poly(lactideco-glycolide-co-caprolactone) for prostate cancer treatment: formulation, characterization and cytotoxicity studies. Nanoscale Res Lett. 2011; 6(1):260.

20. Sanna V, Lubinu G, Madau P, et al. Polymeric nanoparticles encapsulating white tea extract for nutraceutical application. J Agric Food Chem. 2015;63(7):2026-2032.

21. Adhami VM, Malik A, Zaman N, et al. Combined inhibitory effects of green tea polyphenols and selective cyclooxygenase- 2 inhibitors on the growth of human prostate cancer cells both in vitro and in vivo. Clin Cancer Res. 2007;13(5):1611-1619.

22. Siddiqui IA, Malik A, Adhami VM, et al. Green tea polyphenol EGCG sensitizes human prostate carcinoma LNCaP cells to TRAIL-mediated apoptosis and synergistically inhibits biomarkers associated with angiogenesis and metastasis. Oncogene. 2008;27(14):2055-2063.

23. Siddiqui IA, Bharali DJ, Nihal M, et al. Excellent anti-proliferative and pro-apoptotic effects of (-)-epigallocatechin-3-gallate encapsulated in chitosan nanoparticles on human melanoma cell growth both in vitro and in vivo. Nanomedicine. 2014;10(8):1619-1626.

24. Siddiqui IA, Adhami VM, Bharali DJ, et al. Introducing nanochemoprevention as a novel approach for cancer control: Proof of principle with green tea polyphenol epigallocatechin-3-gallate. Cancer Res. 2009; 69(5):1712-1716.

25. Siddiqui IA, Adhami VM, Ahmad N, Mukhtar H. Nanochemoprevention: sustained release of bioactive food components for cancer prevention. Nutr Cancer. 2010;62(7):883-890.

26. Leonarduzzi G, Testa G, Sottero B, Gamba P, Poli G. Design and development of nanovehicle-based delivery systems for preventive or therapeutic supplementation with flavonoids. Curr Med Chem. 2010; 17(1):74-95.

27. Siddiqui IA, Mukhtar H. Nanochemoprevention by bioactive food components: a perspective. Pharm Res. 2010;27(6):1054-1060.

28. Sanna V, Pintus G, Roggio AM, et al. Targeted biocompatibile nanoparticles for the delivery of (-)-epigallocatechin 3-gallate to prostate cancer cells. J Med Chem. 2011;54(5):1321-1332.

29. Sanna V, Sechi M. Nanoparticle therapeutics for prostate cancer treatment. Nanomedicine. 2012;8 Suppl 1:S31-S36.

30. Sanna V, Sechi M. Nanoparticle therapeutics for prostate cancer treatment. Maturitas. 2012;73(1):27-32.

31. Siddiqui IA, Adhami VM, Chamcheu JC, Mukhtar H. Impact of nanotechnology in cancer: emphasis on nanochemoprevention. Int J Nanomedicine. 2012;7:591-605.

32. Sanna V, Siddiqui IA, Sechi M, Mukhtar H. Nanoformulation of natural products for prevention and therapy of prostate cancer. Cancer Lett. 2013;334(1):142-151.

33. Surh YJ. Cancer Chemoprevention with dietary phytochemicals. Nat Rev Cancer. 2003;3(10):768-780.

34. Reddy L, Odhav B, Bhoola KD. Natural products for cancer prevention: a global perspective. Pharmacol Ther. 2003;99(1):1-13.

35. Bettuzzi S, Brausi M, Rizzi F, Castagnetti G, Peracchia G, Corti A. Chemoprevention of human prostate cancer by oral administration of green tea catechins in volunteers with high-grade prostate intraepithelial neoplasia: a preliminary report from a one-year proof-of-principle study. Cancer Res. 2006;66(2):1234-1240.

36. Kurahashi N, Sasazuki S, Iwasaki M, Inoue M, Tsugane S; JPHC Study Group. Green tea consumption and prostate cancer risk in japanese men: a prospective study. Am J Epidemiol. 2008;167(1):71-77. 
37. Khan N, Adhami VM, Mukhtar H. Review: green tea polyphenols in chemoprevention of prostate cancer: preclinical and clinical studies. Nutr Cancer. 2009;61(6):836-841.

38. Mukhtar H. Chemoprevention: Making it a success story for controlling human cancer. Cancer Lett. 2012;326(2):123-127.

39. Millimouno FM, Dong J, Yang L, Li J, Li X. Targeting apoptosis pathways in cancer and perspectives with natural compounds from mother nature. Cancer Prev Res (Phila). 2014;7(11):1081-107.

40. Byrne JD, Betancourt T, Brannon-Peppas L. Active targeting schemes for nanoparticle systems in cancer therapeutics. Adv Drug Deliv Rev. 2008;60(15):1615-1626.

41. Alexis F, Pridgen E, Molnar LK, Farokhzad OC. Factors affecting the clearance and biodistribution of polymeric nanoparticles. Mol Pharm. 2008;5(4):505-515.

42. Sanna V, Pala N, Sechi M. Targeted therapy using nanotechnology: focus on cancer. Int J Nanomedicine. 2014;9:467-483.

43. Huang Y, Zhou D, Hang T, et al. Preparation, characterization, and assessment of the antiglioma effects of liposomal celastrol. Anticancer Drugs. 2012;23(5):515-524.

44. Elzein T, Nasser-Eddine M, Delaite C, Bistac S, Dumas P. FTIR study of polycaprolactone chain organization at interfaces. J Colloid Interface Sci. 2004;273(2):381-387.

45. Kister G, Cassanas G, Bergounhon M, Hoarau D, Vert M. Structural characterization and hydrolytic degradation of solid copolymers of d,1-lactide-co- $\varepsilon$-caprolactone by Raman spectroscopy. Polymer. 2000; 41(3):925-932.

46. Ermeydan MA, Cabane E, Hass P, Koetz J, Burgert I. Fully biodegradable modification of wood for improvement of dimensional stability and water absorption properties by poly( $\varepsilon$-caprolactone) grafting into the cell walls. Green Chem. 2014;16:3313-3321.
47. Zhang X, Zhang T, Zhou X, et al. Enhancement of oral bioavailability of tripterine through lipid nanospheres: preparation, characterization, and absorption evaluation. J Pharm Sci. 2014;103(6):1711-1719.

48. Fonseca C, Simoes S, Gaspar R. Paclitaxel-loaded PLGA nanoparticles: preparation, physicochemical characterization and in vitro anti-tumoral activity. J Control Release. 2002;83(2):273-286.

49. Panyam J, Labhasetwar V. Dynamics of endocytosis and exocytosis of poly(D,L-lactide-co-glycolide) nanoparticles in vascular smooth muscle cells. Pharm Res. 2003;20(2):212-220.

50. Siddiqui IA, Sanna V, Ahmad N, Sechi M, Mukhtar H. Resveratrol nanoformulation for cancer prevention and therapy. Ann N Y Acad Sci. 2015;1348(1):20-31.

51. Zhou YX, Huang YL. Antiangiogenic effect of celastrol on the growth of human glioma: an in vitro and in vivo study. Chin Med J (Engl). 2009;122(14):1666-1673.

52. Pang X, Yi Z, Zhang J, et al. Celastrol suppresses angiogenesis-mediated tumor growth through inhibition of AKT/mammalian target of rapamycin pathway. Cancer Res. 2010;70(5):1951-1959.

53. Khan N, Afaq F, Mukhtar H. Cancer chemoprevention through dietary antioxidants: progress and promise. Antioxid Redox Signal. 2008; 10(3):475-510

54. Rouleau M, Patel A, Hendzel MJ, Kaufmann SH, Poirier GG. PARP inhibition: PARP1 and beyond. Nat Rev Cancer. 2010;10(4):293-301.

55. Shao L, Zhou Z, Cai Y, et al. Celastrol suppresses tumor cell growth through targeting an AR-ERG-NF- $\kappa$ B pathway in TMPRSS2/ERG fusion gene expressing prostate cancer. PLoS One. 2013;8(3):e58391.
International Journal of Nanomedicine

\section{Publish your work in this journal}

The International Journal of Nanomedicine is an international, peerreviewed journal focusing on the application of nanotechnology in diagnostics, therapeutics, and drug delivery systems throughout the biomedical field. This journal is indexed on PubMed Central, MedLine, CAS, SciSearch ${ }^{\circledR}$, Current Contents ${ }^{\circledR} /$ Clinical Medicine,

\section{Dovepress}

Journal Citation Reports/Science Edition, EMBase, Scopus and the Elsevier Bibliographic databases. The manuscript management system is completely online and includes a very quick and fair peer-review system, which is all easy to use. Visit http://www.dovepress.com/ testimonials.php to read real quotes from published authors. 